\title{
130 nm CMOS Fully Differential SC Filter for Ultra-Low Voltage $\Sigma-\Delta$ Converter
}

\author{
David Maljar, Daniel Arbet and Viera Stopjaková \\ Institute of Electronics and Photonics \\ Faculty of Electrical Engineering and Information Technology \\ Slovak University of Technology \\ Email: daniel.arbet@stuba.sk
}

\begin{abstract}
In this paper design and function of the fully differential (FD) switched-capacitor (SC) integrator for ultra-low voltage Sigma-Delta analog to digital converter $(\Sigma-\Delta$ ADC) are presented. The proposed integrator was designed for differential input signal and applicable as a main analog block of ultra-low voltage $\Sigma-\Delta$ ADC in standard $130 \mathrm{~nm}$ CMOS technology. The main block of proposed integrator is operational transconductance amplifier (OTA) based on two-stage Rail-to-Rail (RtR) FD operational amplifier (OPAMP) working in sub-threshold regime. The characteristic properties of this circuit is non-standard OTA topology, using SC common-mode feedback (CMFB) circuit and using switching T-gates. All of these subcircuits are supplied by only $0.6 \mathrm{~V}$ with achieved gain $24.09 \mathrm{~dB}$ and cutoff frequency $165.95 \mathrm{kHz}$.
\end{abstract}

Keywords-analog design, ultra-low voltage, switchedcapacitor integrator, Sigma-Delta ADC, CMOS

\section{INTRODUCTION}

Nowadays, analog designers are facing difficult challenges due to evolution of CMOS nanometer technology and continuous scaling-down of the power supply voltage of integrated circuits (IC). These things cause decreasing dimensions of transistors and affect to the properties of the analog circuits. Usage of transistors as resistors, capacitors, amplifiers or current sources is still needed in analog design but the requirement to minimize the supply voltage brings many problems. For example, more than 2 or 3 transistors cannot be stacked because of the voltage headroom issue. In addition, many of them work in weak inversion region where current $I_{D}$ exponentially depends on voltage $V_{G S}$ and where is considerable parasitic capacitance effect. On the contrary, in digital design, transistors are mostly used as the switches and there are not so many problems of this kind with them. It is one of the most important advantage of digital ICs.

ADCs has been moved to the CMOS nanometer technologies as an interface between analog and digital circuits. Both of these kinds of circuits are implemented on single chip, so they have to be powersupplied by one $V_{D D}$ voltage. Since the digital part of the circuit can operate with an ultra-low supply voltage, the analogue parts of the circuit are adapted to these ultra-low values of $V_{D D}$ as much as possible. In $\Sigma-\Delta$ ADC is low-pass filter - integrator the main block of the analog part of the circuit. It causes the pulses to densify on the digital output signal.
Compared to the input, the shape of the signal is different, but its nature is preserved [1], [2], [3].

Fig. 1 shows differential block scheme of $\Sigma-\Delta$ ADC. In this scheme, analog input signal is compared (by difference amplifiers) with reference signal coming from 1 - bit digital to analog converters (DACs). This modified signal is then integrated and compared to the reference voltage in comparators (in generally $0 \mathrm{~V})$. Then looped to 1-bit DACs whose outputs are changing mentioned analog input reference in the difference amplifiers. D-type flip-flops are used for synchronization of digital output signal [4].

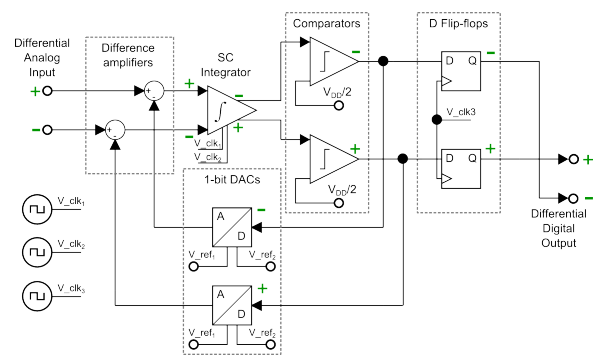

Fig. 1. The differential first-order signle-loop output-synchronized $\Sigma-\Delta$ ADC

In this paper, the principle of the integrator design is introduced in section II. The achieved results obtained from simulations are stated in section III and the evaluation of all process is summarized in the last section IV.

\section{PROPOSED INTEGRATOR}

Topology of FD SC integrator block from Fig. 1 is introduced in the Fig. 2.



Fig. 2. Topology of FD SC integrator 


\section{A. Operational transconductance amplifier - OTA}

The topology of OTA is shown in the Fig. 3. This circuit is based on parallel connection of two-stage NMOS and PMOS OPAMPs working in sub-threshold regimes.

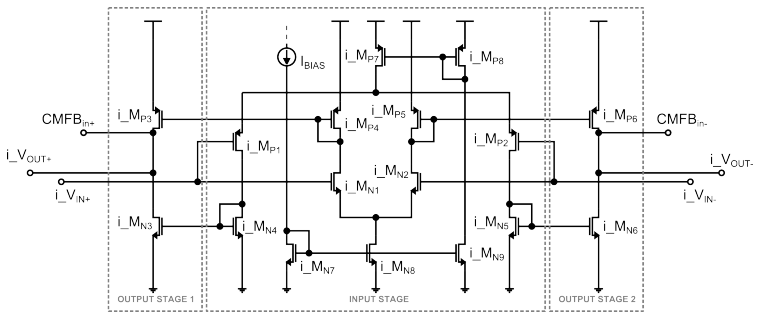

Fig. 3. Topology of OTA

Complementary connection of NMOS and PMOS in the OPAMP output stage allows RtR operation at output. It is well-know that input voltage range of PMOS transistor is limited from above by the voltage:

$$
V_{P M O S_{M A X}}=V_{D D}-\left|V_{D S_{-} s a t}\left(i_{-} M_{P 7}\right)\right|-\left|V_{T H}\left(i_{-} M_{P 1}\right)\right|
$$

In the range from $V_{P M O S_{M A X}}$ to $V_{D D}$ is NMOS transistor active only. Contrariwise, the input voltage range of NMOS transistor is limited from below by the voltage:

$$
V_{N M O S_{M I N}}=V_{D S \_s a t}\left(i_{-} M_{N 8}\right)+V_{T H}\left(i_{-} M_{N 1}\right)
$$

In the range from ground to $V_{N M O S_{M I N}}$ is PMOS transistor active only.

Around the $\frac{V_{D D}}{2}$ voltage, both of transistors are in the active region. In the area where both amplifiers operate in the active region, the transconductances $g_{m \_N M O S}$ and $g_{m_{-}} P M O S$ are overlapped. In general, by increasing $g_{m}$, the dominant pole is shifted towards higher frequencies. This may result in frequency response and overall circuit stability. Transistors $i_{-} M_{N 1}, i_{-} M_{N 2}, i_{-} M_{P 1}$ and $i_{-} M_{P 2}$ have been designed with $g_{m}$ compensation as is shown in Fig. 4:

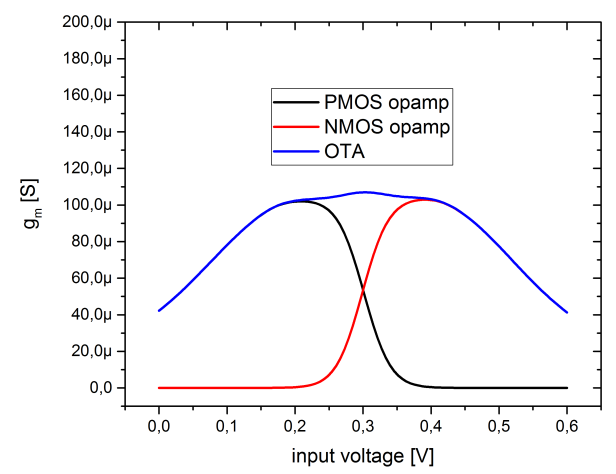

Fig. 4. Compensation of $g_{m_{-} P M O S}$ and $g_{m_{-} N M O S}$
The input transistors $\left(i_{-} M_{N 1}, i_{-} M_{N 2}, i_{-} M_{P 1}\right.$, $i_{-} M_{P 2}$ ) was set to the sub-treshold region by the equations:

$$
\begin{gathered}
V_{e f f}=2 \cdot V_{T} \cdot n \cdot \ln (\exp (\sqrt{I F})-1) \\
\frac{W}{L}=\frac{I_{D}}{I_{D 0} \cdot \exp \left(\frac{V_{e f f}}{n \cdot V_{T}}\right)} .
\end{gathered}
$$

The current sources $\left(i \_M_{P 7}, i_{-} M_{N 8}\right)$ works in strong saturation by the $\bar{V}_{D S}$ voltage approximately $100 \mathrm{mV}$. Transistors $i_{-} M_{N 7}, i_{-} M_{N 9}$ and $i_{-} M_{P 8}$ was used as the simple current mirrors for circuit biasing. $i_{-} M_{P 4}, i_{-} M_{P 5}, i_{-} M_{N 4}$ and $i_{-} M_{N 5}$ was used as a current mirrors for output stage 1 and 2 [5].

As an output stage, AB class amplifier is used. Dimensions of the transistors $i_{-} M_{P 3}, i_{-} M_{N 3}, i_{-} M_{P 6}$ and $i_{-} M_{N 6}$ was designed for relatively high currents in that branches due to driving output capacitors and capacitors in CMFB circuit.

\section{B. Clock-boost circuit}

In order to control switching transistors in CMFB circuit clock-boost circuit was used. Its topology is shown in the Fig. 5. Two inverters at the beginning of the circuit modify the rising and the falling edges of the clock signal for better operate with. Cross-coupled NMOS transistors $b{ }_{-} M_{N 1}$ and $b \_M_{N 2}$ together with the capacitors $b \_C_{1}$ and $b \_C_{2}$ are forming a charge pump. Pulses from the clock are shifted up by adding $V_{D D}$ voltage to them. By the transistors $b_{-} M_{N 3}$ and $b \_M_{N 4}$ is this shifted voltage (in the other words: shifted charge) connected to a "bootstrap" capacitor $b \_C_{3}$. By switching the transistors $b_{-} M_{P 1}, b M_{N 5}$, $b \_M_{P 2}, \quad b \_M_{N 6}, \quad b \_M_{N 7}, \quad b \_M_{N 8}$ and $b \_M_{N 9}$ is mentioned charge transported to the output - switching transistor in SC CMFB circuit. This circuit was designed as a part of SC CMFB circuit as a "bootstrap" [2].

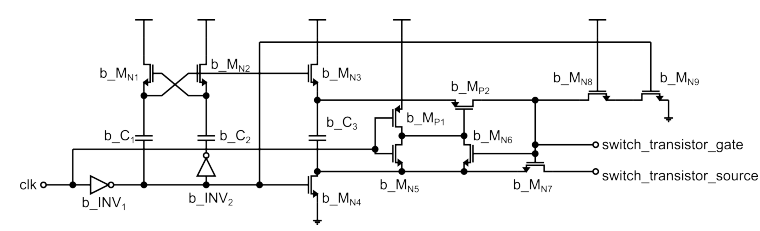

Fig. 5. Topology of clock-boost circuit

\section{Switched-capacitor CMFB circuit}

Fig. 6 shows the topology of the SC CMFB circuit. There are three task for CMFB circuit: sensing the common mode outputs $i_{-} V_{O U T+}$ and $i_{-} V_{O U T-}$ from fully differential OTA, comparison of the sensed results with a reference voltage $V_{C M}$ (in this design, the reference voltage for CMFB circuit is equal to $\frac{V_{D D}}{2}$ ) and return the bias voltage $V_{B I A S}$ back to the OTA by $C M F B$ output. This SC CMFB circuit is clocked by non-overlapping pulses with frequency $f_{s w}=20 \mathrm{MHz}$. These pulses are shifted up in "bootstrap" circuits for better gate-switching of all the 
transistors in the Fig. 6 [2]. All the transistors have been designed with the same dimensions as a switches with a low enough $R_{O N}$ parameter. The capacitors $c m_{-} C_{1}\left(c m \_C_{2}\right)$ and $c m \_C_{3}\left(c m \_C_{4}\right)$ have been designed with a specific ratio $k$ :

$$
k=\frac{c m \_C_{1}}{c m \_C_{3}}=\frac{c m \_C_{2}}{c m \_C_{4}}
$$

In order to obtain low value of output offset, the specific ratio capacitors $\mathrm{cm} \mathrm{C}_{1}\left(\mathrm{~cm} \mathrm{C}_{2}\right)$ and $\mathrm{cm}_{-} \mathrm{C}_{3}$ $\left(\mathrm{cm} \mathrm{C}_{4}\right)$ should be perfectly set. However, $\mathrm{cm} \mathrm{C}_{-} \mathrm{C}_{1}$ and $\mathrm{cm}_{-} \mathrm{C}_{2}$ can not be too large, because OTA will not be able to drive them. On the contrary, $\mathrm{cm}_{-} C_{3}$ and $\mathrm{cm}_{-} \mathrm{C}_{4}$ can not be too small, because the parasitic capacitance of the switching transistors will start to affect the circuit. Transistors $c m_{-} M_{P 1}, c m_{-} M_{P 2}$, $\mathrm{cm} \mathrm{M}_{N} M_{N}$ and $\mathrm{cm} \mathrm{M}_{-} M_{N 10}$ form the output invertors for driving the SC CMFB outputs. These outputs are connected to the OTA, as it is shown in the Fig. 2 and Fig. 3.

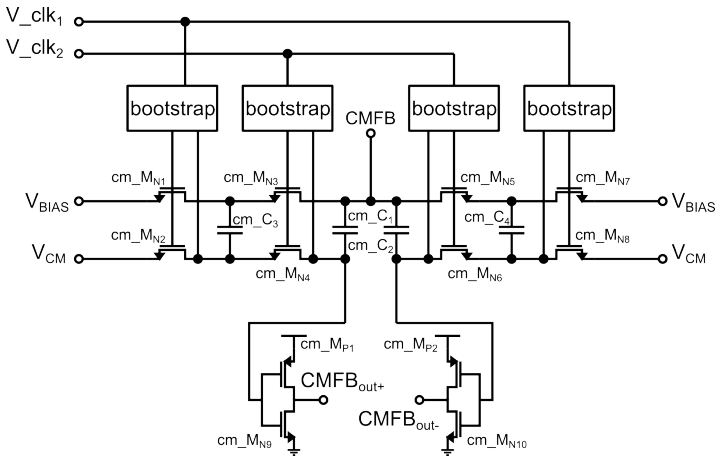

Fig. 6. Topology of switched-capacitor CMFB circuit

\section{Switching transmission gates (T-gates)}

As it is shown in the Fig 2., capacitors $i_{-} C_{S 1}-$ $i_{-} C_{S 4}$ are switched with transmission gates (T-gates) $i_{-} T_{1}-i_{-} T_{8}$ for better charge transport. For initial values of capacitors, voltage gain and cutoff frequency the following equations can be used:

$$
\begin{gathered}
C_{s w} \propto \frac{1}{f_{s w} \cdot R_{e q}} \\
A_{I N T_{-} M A X} \propto \frac{i_{-} C_{S 1}}{i_{-} C_{S 3}} \\
f_{\text {cutoff }}=B W_{I N T} \propto \frac{1}{2 . \pi \cdot i_{-} C_{1} \cdot R_{e q}\left(i_{-} C_{S 3}\right)}
\end{gathered}
$$

\section{ACHIEVED RESULTS}

The integrator has been simulated with the signals of varying frequencies, amplitudes and types. For DC simulation of the OTA (with disconnected SC CMFB circuit), the input voltage was swept in the range of $G N D$ and $V_{D D}$. In the $\mathrm{AC}$ simulation, two periodic sources with $180^{\circ}$ phase shift were used for simulate differential input signal. Because of using clocks, periodic steady-state analysis (PSS analysis) and periodic AC analysis (PAC analysis) was used. In the Fig. 7 are shown the transfer responses of inverting and non-inverting outputs, where RtR output voltage range can be obtained. Fig. 8 shows frequency response of OTA with maximum gain of $A_{O T A \_M A X}=46.16 \mathrm{~dB}$. Bandwidth of proposed OTA is $B W_{O T A}=24.23 \mathrm{kHz}$, while gain-bandwidth is $G B W=5.03 \mathrm{MHz}$.

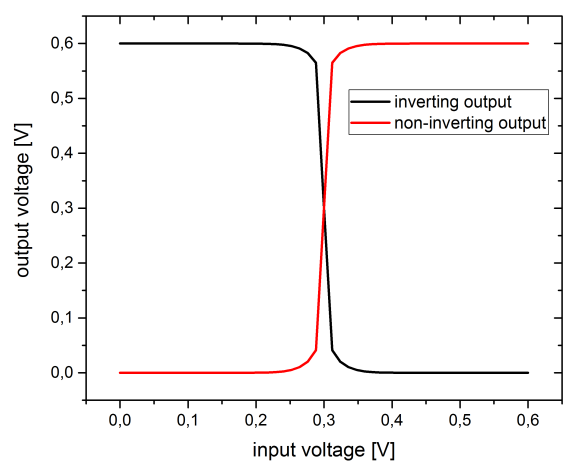

Fig. 7. Transfer response of OTA

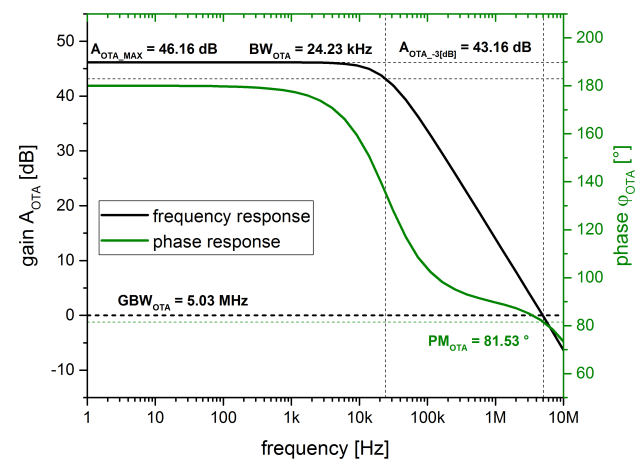

Fig. 8. Frequency and phase response of OTA

Fig. 9 shows frequency response of whole integrator where low-frequency gain of $A_{I N T \_M A X}=24.09 \mathrm{~dB}$ and cutoff frequency $f_{\text {cutoff }}=165.95 \mathrm{kHz}$ were achieved.

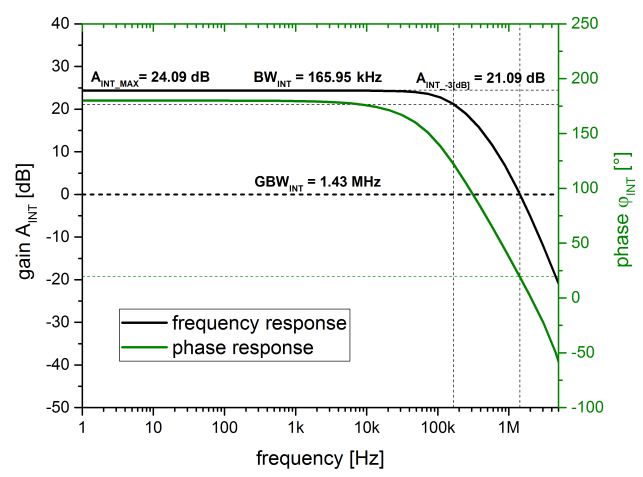

Fig. 9. Frequency and phase response of integrator

In Fig. 10 is shown time response of integrator to a harmonic input signal and Fig. 11 shows time response of integrator to a pulse input signal. 


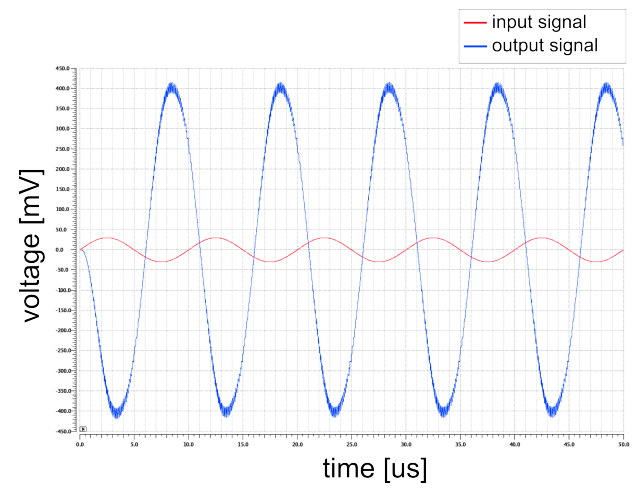

Fig. 10. Harmonic signal response - integration

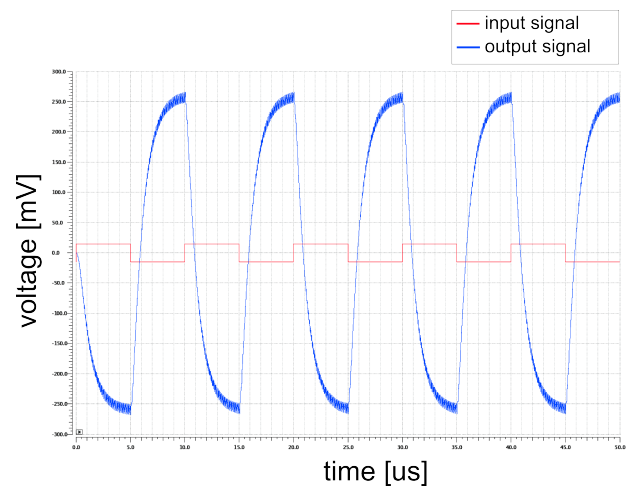

Fig. 11. Pulse signal response - integration

All the essential achieved parameters are summarized in the Tab. 1.

TABLE I. INTEGRATOR PARAMETERS

\begin{tabular}{l|c} 
Parameter & Value \\
\hline \hline$V_{D D}$ & $0.6 \mathrm{~V}$ \\
\hline$C_{L O A D}$ & $10 \mathrm{pF}$ \\
\hline$A_{O T A_{-} M A X}$ & $46.16 \mathrm{~dB}$ \\
\hline$B W_{O T A}$ & $24.23 \mathrm{kHz}$ \\
\hline$G B W_{O T A}$ & $5.03 \mathrm{MHz}$ \\
\hline$P M_{O T A}$ & $81.53^{\circ}$ \\
\hline$A_{I N T \_M A X}$ & $24.09 \mathrm{~dB}$ \\
\hline$B W_{I N T}$ & $165.95 \mathrm{kHz}$ \\
\hline$G B W_{I N T}$ & $1.43 \mathrm{MHz}$ \\
\hline$P_{I N T}$ & $22.16 \mu \mathrm{W}$ \\
\hline
\end{tabular}

In addition, interesting thing is the effect of the dimensions of transistor to shape of the switched signal. In the Fig. 12 is the signal switched by NMOS transistor of small dimensions.

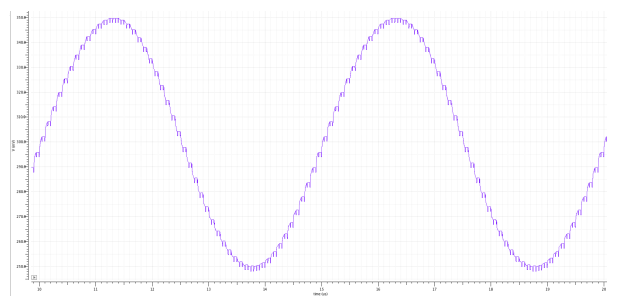

Fig. 12. Switched signal - W and L small enough
In the Fig. 13 is this signal switched by NMOS transistor with the same width as in the Fig. 12, but with increased length.

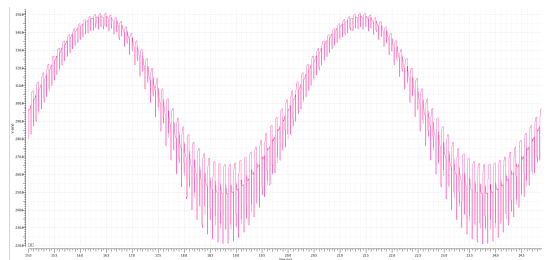

Fig. 13. Switched signal - small W, larger L

In the Fig. 14 is this signal switched by NMOS transistor with the same length as in the Fig. 12, but with increased width.

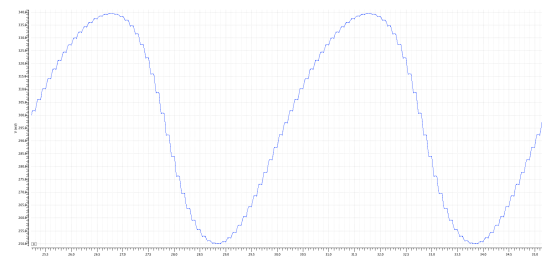

Fig. 14. Switched signal - small L, larger W

\section{CONCLUSION}

Ultra low-voltage RtR fully differential switchedcapacitor filter with supply voltage of $0.6 \mathrm{~V}$ was designed for load capacitance $10 \mathrm{pF}$. Power consumption of this circuit is $22.16 \mu \mathrm{W}$. If necessary, gain of the integrator $24.09 \mathrm{~dB}$ can be increased by larger ratio of capacitors $i_{-} C_{S 1}$ and $i_{-} C_{S 3}$. It is important to note, that for possibly increase the gain, it is necessary to design output stages of OTA for higher current values.

This switched-capacitor integrator will be used as the main part of currently designing Sigma-Delta analog to digital converter. Mentioned converter will be developed for energy-harvesting applications (solar panels, wind power stations etc.).

\section{ACKNOWLEDGMENT}

This work was supported in part by the Ministry of Education, Science, Research and Sport of the Slovak Republic under grant VEGA 1/0731/20, ECSEL JU under project PROGRESSUS (876868) and by the Slovak Research and Development Agency under grant APVV 19-0392.

\section{REFERENCES}

[1] N. Gandhi and S. Shelke, "Sigma delta analog to digital converter: Design and implementation with reduction in power consumption," pp. 1035-1039, 052017.

[2] L. Yao, M. Steyaert, and W. Sansen, Low-power low-voltage Sigma-Delta modulators in nanometer CMOS. Springer, 2006.

[3] Lee, Song, and Roh, "A 103 dB DR Fourth-Order DeltaSigma Modulator for Sensor Applications," Electronics, vol. 8, p. 1093, 092019.

[4] R. Schreier, G. C. Temes, and S. Pavan, Understanding DeltaSigma data converters. Hoboken, New Jersey: John Wiley and sons, Inc., 2nd ed., 2017.

[5] B. Razavi, Design of analog CMOS integrated circuit. McGraw Hill, 1st ed., 2001. 